\title{
Esporotricosis: la micosis subcutánea más distribuida en el mundo
} Sporotrichosis: the most widely distributed subcutaneous mycosis in the world

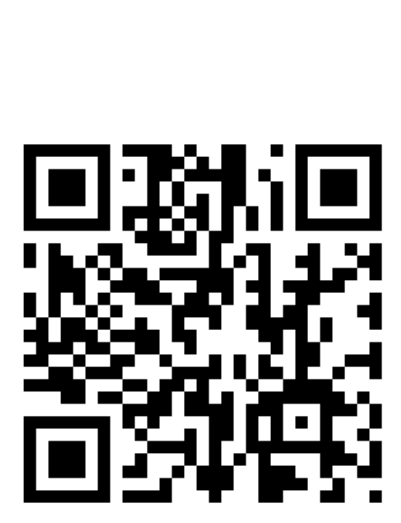

Recibido 03/08/2021

\author{
${ }^{1}$ Dr. José Roberto Hernández Chacón \\ Hospital Nacional de Geriatría y Gerontología, San José, Costa Rica \\ (D) https://orcid.org/0000-0002-1174-4887 \\ ${ }^{2}$ Dra. Sheirys Arleth Torres Morales \\ Hospital San Juan de Dios, San José, Costa Rica \\ (iD https://orcid.org/0000-0003-0134-8175
}

${ }^{3}$ Dra. Evelyn María Hernández Chacón Centro de Desarrollo Estratégico e Información en Salud y Seguridad Social (CENDEISSS), San José, Costa Rica

(D) https://orcid.org/0000-0001-5592-5703
Corregido $11 / 08 / 2021$
Aceptado

$20 / 08 / 2021$

\section{RESUMEN}

La esporotricosis es una enfermedad micótica causada por hongos de la familia Sporothrix schenckii, puede cursar de manera subaguda o crónica y afecta principalmente al tejido subcutáneo y linfático. La transmisión se realiza principalmente mediante la inoculación con material contaminado en zonas de la piel que tienen un trauma. Puede afectar tanto a hombres como a mujeres y es más común en pacientes pediátricos y adultos jóvenes. Es la micosis por implantación más distribuida en el mundo, se puede encontrar tanto en regiones intertropicales como en climas templados. Presenta varias formas clínicas, la más frecuente es la linfocutánea que se puede encontrar en un $70 \%$ de los casos, seguido por la forma fija en un $25 \%$. El diagnóstico se realiza mediante biopsia del nódulo para poder realizar el cultivo micológico y confirmar la sospecha diagnóstica. El fármaco de elección contra las formas cutáneas linfangítica y fija es itraconazol, pero tiene la desventaja de ser un medicamento de alto costo, por lo que el tratamiento de elección en países en vías de desarrollo ha sido el yoduro de potasio, ya que se caracteriza por ser efectivo, bien tolerado y de bajo costo.

PALABRAS CLAVE: esporotricosis; Sporothrix sp; micosis.

\section{ABSTRACT}

Sporotrichosis is a fungal disease caused by the fungi of the Sporothrix schenckii family, it can occur subacute or chronic and mainly affects the subcutaneous and lymphatic tissue. 
Transmission is carried out mainly by inoculation with contaminated material in areas of the skin that have a trauma. It affects men and women and is most common in pediatric and young adults patients. It is most widely distributed mycosis due to implantation in the world, it can be found in intertropical regions and in temperate climates. It presents several clinical forms, the most frequent is the lymphocutaneous form, that can be found in $70 \%$ of the cases, followed by the fixed form in $25 \%$. The diagnosis is made by biopsy of the nodule to be able to carry out the mycological culture and confirm the diagnostic suspicion. The drug choice against the fixed and lymphangitic cutaneous forms is itraconazole, but it has the disadvantage of being a high-cost drug. The treatment in developing countries has been potassium iodide, since it is characterized by being effective, well tolerated, and low price.

KEYWORDS: sporotrichosis; Sporothrix sp; mycosis.

${ }^{1}$ Médico general, graduado de la Universidad de Iberoamérica de Costa Rica (UNIBE). Cód. MED14016. Correo: robhernandezch@gmail.com

${ }^{2}$ Médica general, graduada de la Universidad de Iberoamérica de Costa Rica (UNIBE). Cód. MED14370. Correo: sheirystorres@gmail.com

${ }^{3}$ Médica general, graduada de la Universidad de Iberoamérica de Costa Rica (UNIBE). Cód. MED16718. Correo: evelmhch@gmail.com

\section{INTRODUCCIÓN}

La enfermedad se descubrió en 1898, en el hospital de Johns Hopkins en Baltimore Maryland, cuando un estudiante de Medicina llamado Benjamin Schenk, identificó por primera vez la esporotricosis en un hombre de 36 años quien presentaba lesiones en la mano y el brazo derecho. Tiempo después, en el año 1900 se denominó el hongo causal de dichas lesiones Sporothrix schenckii, por Hektoen y Perkins (1).

La esporotricosis es una enfermedad micótica causada por hongos de la familia Sporothrix schenckii (S. schenckii), se han identificado seis especies causantes de la enfermedad, las tres más frecuentes son $S$. schenckii, Sporothrix brasiliensis (S. brasiliensis) y Sporothrix globosa (2). Dicha enfermedad puede cursar de manera subaguda o crónica y afecta principalmente al tejido subcutáneo y el tejido linfático; sin embargo, puede afectar tejidos profundos como los huesos, articulaciones, pulmón y cerebro $(3,4)$. Es la micosis por implantación más distribuida en el mundo, se puede encontrar tanto en regiones intertropicales como en climas templados, se puede encontrar en diversos países de centroamérica como en México, Guatemala y Costa Rica; en Sudamérica como en los países de Colombia, Uruguay, Brasil y Perú, en donde presenta una incidencia anual estimada de 48 a 60 casos por 100000 habitantes (5); además de países del continente africano y asiático $(2,3,6)$.

Las especies viven en el suelo, materia orgánica, plantas, paja, ramas, zacate y musgos. La transmisión se realiza mediante la inoculación con material contaminado en zonas de la piel que tienen un trauma, por ese motivo los grupos de alto riesgo para adquirir la enfermedad son los trabajadores de granjeros, viveros, horticultores, jardinería, carpinteros y amas de casa que trabajan sus jardines $(2,3)$. La enfermedad también puede transmitirse como una enfermedad zoonótica, por ejemplo, a través de los perros, gatos, loros, burros, armadillos, caballos, entre otros $(3,7)$. Muchos han sido diagnosticados como portadores asintomáticos de Sporohtrix sp. y la transmisión a humanos se ha dado a través de mordedura y/o contacto abrasivo (8). La esporotricosis puede presentarse a 
cualquier edad, sin embargo, es más común en pacientes pediátricos y adultos jóvenes de 16 a 35 años. Se han descrito factores de riesgo de enfermarse, independientemente de la actividad laboral, tales como el alcoholismo, la diabetes mellitus, EPOC (enfermedad pulmonar obstructiva crónica), uso de medicamentos inmunosupresores, neoplasias hematológicas, entre otros (1).

Una forma de transmisión poco común es por medio de inhalación del hongo, el cual viaja a través del tracto respiratorio superior, de esta manera puede desarrollarse una neumonitis granulomatosa y diseminación hematógena. En este caso debe diferenciarse de una infección por tuberculosis (1).

Al tratarse de una enfermedad con una baja incidencia, pero que, a la vez, es la micosis por implantación más distribuida en el mundo, se realiza una revisión bibliográfica que permite valorar los artículos y estudios más recientes, con el objetivo de mantener actualizado a los profesionales de la salud sobre la esporotricosis, dando énfasis en el diagnóstico y tratamiento adecuado.

\section{MÉTODO}

Se realiza una revisión bibliográfica de artículos y de estudios científicos relevantes, que den validez a lo planteado en el objetivo de esta investigación. Se buscaron estudios en los idiomas inglés y español, y publicados entre los años 2015 y 2021. Se utilizaron buscadores tales como Cochrane Database, Scientific Electronic Library Online (SciELO) y Medical Literature Analysis and Retrieval System Online (MEDLINE / PUBMED). Los descriptores empleados fueron "esporotricosis", "esporotricosis linfocutánea", "esporotricosis fija", "micosis" y "Sporothrix sp." Para la selección de las publicaciones se analizó el texto completo y se identificaron los más relevantes para desarrollar el artículo. Al final del proceso de selección se escogieron 15 artículos.

\section{FORMAS CLÍNICAS Y PATOGENIA}

Presenta varias formas clínicas, en personas inmunocompetentes la más frecuente es la linfocutánea que se puede encontrar en un $70 \%$ de los casos y se presenta con lesiones nodulares eritematosas y violáceas que en ocasiones se pueden ulcerar drenando un material purulento, esta zona se denomina chancro de inoculación y posteriormente se desarrollan lesiones nodulares lineales siguiendo el trayecto linfático $(1,6,9)$. En esta presentación se afectan principalmente las extremidades y la cara; generalmente es unilateral. La sintomatología puede abarcar prurito y dolor local e incluso en ciertos casos puede existir linfedema cuando el drenaje linfático se afecta (1).

La forma fija se presenta en un $25 \%$ de los casos y pueden ser lesiones vegetantes o verrugosas en el sitio de inoculación. En personas inmunocomprometidas también suelen afectarse otros órganos, la forma clínica puede variar dependiendo al estado inmunológico del hospedero, de la carga fúngica, la profundidad y del lugar de la inoculación $(9,10)$. Otra forma de presentación es la nocardiosis cutánea que se manifiesta como abscesos, pústulas, piodermas, granulomas y celulitis (11).

La esporotricosis se clasifica en lesiones cutáneas y extracutáneas, entre la primera se encuentran la linfática, fija y diseminada; y entre la segunda se encuentran la pulmonar, visceral y osteoarticular, entre otras (12). Se deben diferenciar de otras enfermedades que pueden presentar lesiones cutáneas similares, tales como la sarcoidosis, rosácea, carcinoma basocelular, carcinoma epidermoide, 
queratoacantoma, sarcoma y pioderma gangrenoso (2).

Cuando el hongo se encuentra en el órgano diana, inicia una reacción granulomatosa supurativa en los tejidos, siendo la inmunidad de células $\mathrm{T}$ dependientes de óxido nítrico muy importante para lograr el control de la enfermedad (1).

Los factores de virulencia son moléculas o estructuras de los hongos que les permiten permanecer y proliferar dentro del organismo que han parasitado. La quitina es el componente en menor cantidad en las diversas formas de vida del hongo, mientras que la b-glucanas y ramnomananas son las más abundantes (8).

Las especies más virulentas como $S$. brasiliensis y $S$. schenckii, contienen en su genoma 9 y 10 genes respectivamente, que codifican para quitinasas. La producción de melanina depende de la concentración de glucosa en el medio de cultivo y protege al microorganismo de ser fagocitado (8).

Otro factor de virulencia son las integrinas y adhesinas del hongo; además, el ergosterol de la membrana celular y los exoantígenos de la forma de levadura, éstos activan el sistema inmune innato y adquirido del huésped y citoquinas productoras de especies reactivas de oxígeno, dicho proceso resultará en la producción de citoquinas tipo Th1 y Th2, impulsado por la acción de la interleucina (IL) 12 y 4 respectivamente $(13,14)$.

\section{HISTOLOGÍA}

El análisis histopatológico puede orientar un diagnóstico, sin embargo, no es patognomónico. Al analizar la biopsia histológicamente se puede encontrar una zona sifiloide, en donde se pueden encontrar plasmocitos, linfocitos, fibroblastos y una neoformación vascular; además se puede encontrar una zona tuberculoide que se forma por células epitelioides y células gigantes multinucleadas de tipo Langhans; por último, se puede encontrar una zona supurativa crónica, en donde se encuentran microabscesos de polimorfonucleares y cuerpos asteroides $(2,12)$. Se debe tener en cuenta que dichas lesiones también se pueden presentar en otras enfermedades, tales como tuberculosis, otras lesiones micóticas profundas y sífilis (13).

\section{DIAGNÓSTICO}

El diagnóstico se realiza primeramente mediante la epidemiología y la clínica que presenta el paciente, y en segundo lugar la biopsia del nódulo para poder realizar el cultivo micológico y confirmar la sospecha diagnóstica $(9,12)$.

El estándar de oro para poder diagnosticar la esporotricosis es el cultivo, ya que muestra el dimorfismo presente en dichas lesiones. La muestra se cultiva en agar dextrosa Sabouraud, ya sea con antimicrobianos o sin ellos. Al observar al microscopio se pueden observar hifas hialinas delgadas $y$ ramificadas que se agrupan típicamente en formaciones, generando una forma de "pétalos de margarita" o "flores de durazno" (15).

Hay otras formas de diagnóstico, como, por ejemplo, el realizar un examen directo $(\mathrm{KOH})$ o tinciones como el PAS y el Grocott del exudado de las lesiones. Estas formas de diagnóstico no son muy útiles, debido a que las levaduras presentes en las lesiones son muy escasas y pueden ser difíciles de observar (2).

La prueba de intradermorreacción mide la hipersensibilidad del hospedero frente al parásito. Sirve para interpretar si el usuario ha estado o no expuesto a la infección, sin embargo, no funciona como un método 
diagnóstico. La intradermorreacción ha permitido determinar si los usuarios están en condiciones de enfrentar la enfermedad o no; además permite poder predecir la evolución y pronóstico de la enfermedad (15).

\section{TRATAMIENTO}

El pronóstico de la enfermedad es benigno, las lesiones cutáneas pueden ser incapacitantes y las lesiones linfangíticas y fijas pueden rara vez resolver de manera espontánea; sin embargo, de manera poco frecuente puede haber lesiones latentes o inclusos letales. Para los casos en donde se requiere tratamiento, hay guías en donde se detalla el manejo a seguir, como lo es la realizada por la Sociedad de Enfermedades Infecciosas de América publicada en el 2007, la cual recomienda que es necesario realizar un tratamiento a la mayoría de los pacientes $(1-3,12)$.

Se indica que el fármaco de elección contra las formas cutáneas linfangíticas y fija es itraconazol, de 100 a $200 \mathrm{mg}$ al día hasta 2 a 4 semanas posterior a la resolución clínica de la lesión, la cual, de tres hasta seis meses, este tratamiento presenta una efectividad es superior a $90 \%$. Tiene la desventaja de ser un medicamento de alto costo En pacientes que se encuentren inmunocomprometidos o casos con enfermedad diseminada la dosis varía en los días 2,19-22, la cual debe elevarse a 300$400 \mathrm{mg}$ al día (1).

Otro tratamiento que en la literatura muestra presentar una buena efectividad para ser un medicamento alternativo al itraconazol a dosis de $200 \mathrm{mg}$ dos veces al día. También una alternativa para los pacientes no lo toleren 0 que presenten interacciones farmacológicas es la terbinafina a dosis de $500 \mathrm{mg}$ dos veces al día $(1,12)$.
El tratamiento de elección en países en vías de desarrollo ha sido el yoduro de potasio, ya que se caracteriza por ser efectivo, bien tolerado y de bajo costo (16); sin embargo, puede provocar algunos efectos colaterales importantes. La dosis recomendada de yoduro de potasio es iniciar con cinco gotas tres veces al día e ir aumentando la dosis según tolerancia, hasta alcanzar dosis de 40 a 50 gotas tres veces al día $(2,12)$.

Se puede utilizar el fluconazol a dosis de 400 a $800 \mathrm{mg}$ cada día únicamente si no tolera los medicamentos indicados anteriormente. La termoterapia local se suele utilizar como adyuvante para acortar los tiempos de curación, es bastante útil mujeres embarazadas o en el período de lactancia o incluso con pacientes que presentan intolerancia a los antifúngicos $(1,12)$.

\section{CONCLUSIONES}

La esporotricosis es una enfermedad que se presenta de manera infrecuente como una enfermedad diseminada o invasiva, esto genera un impacto importante en la salud pública. La mayoría de las publicaciones, describen a esta enfermedad en zonas de climas tropicales y subtropicales como la nuestra, especialmente aquellos que trabajan en zonas rurales como los campesinos y agricultores ya que el hongo se encuentra en la tierra y en material vegetal fresco y seco. También se considera una enfermedad ocupacional en veterinarios por el riesgo de contagio a través de animales como perros y gatos.

El estándar de oro para el diagnóstico sigue siendo el cultivo de agar dextrosa Sabouraud, siempre tomando en cuenta la epidemiología y la clínica del paciente. El tratamiento de elección es el itraconazol, sin embargo, es un medicamento de alto costo por lo que los países en vías de desarrollo 
no siempre tienen acceso a disponer de él; el yoduro de potasio es el tratamiento de elección en estos casos. Las manifestaciones clínicas de la enfermedad son localizadas, principalmente con lesiones cutáneas que resuelven lentamente y que tienden a ser recurrentes. También se puede presentar en forma diseminada que puede afectar diversos órganos. Se encuentra principalmente en personas inmunocomprometidas tales como pacientes con el virus de inmunodeficiencia humana (VIH), diabetes, neoplasias, alcoholismo, entre otros.

En conclusión, se debe estar alerta para diagnosticar esta enfermedad que es poco conocida y diagnosticada, muy frecuentemente observada en Centroamérica y en especial en nuestro país. Siempre se debe considerar esta patología entre las principales posibles causas de enfermedad en personas con síntomas cutáneos como descritos e incluso en afectación de otros órganos.

\section{Los autores declaran no tener conflicto de interés.}

\section{REFERENCIAS}

1. Gaviria-Giraldo CM, Cardona-Castro N. Esporotricosis y cromoblastomicosis: revisión de la literatura. Ces Med. 2017;31(1):77-91.

2. Ochoa-Reyes J, Ramos-Martínez E, TreviñoRangel R, González GM, Bonifaz A. Esporotricosis del pabellón auricular. Comunicación de un caso atípico simulando una celulitis bacteriana. Rev Chil Infectol. 2018;35(1):83-7.

3. Iole S, Belangero N, Teixeira F, Mirlene CSP, Emanuel BS, Melaragno MI. Esporotricosis. Presentación de un caso en Brasil. Soc Bras Cardeología [Internet]. 2019;5(3):29-30. Disponible en: http://scielo.sld.cu/pdf/ric/v98n3/1028-9933-ric98-03-405.pdf

4. Jorge Mayorga-Rodríguez JLM-G, Víctor Fernando Muñoz-Estrada RMDLR.
Esporotricosis: serie de 1134 casos en una zona endémica de Mexico. Med Cutan Ibero Lat Am. 2018;47(1):24-8.

5. Ventura-Flores $\mathrm{R}$, Guzman-Velasquez JM, Tarrillo-Dávila MÁ. Sporotrichosis: An occupational and neglected disease. Rev Cuba Salud Publica. 2018;44(3):3-6.

6. Oyarce JA, García C, Alave J, Bustamante B. Epidemiological clinical and laboratory characterization of sporotrichosis in patients of a tertiary care hospital in Lima, Peru, from 1991 to 2014. Rev Chil infectología. 2016;33(3):315-21.

7. Olivera-cardozo $M$, Pérez-ortiz $V$, Piñón-gámez $A$, Reyna-villasmil ME. Infeccion es fúngicas cutáneas profundas: Revisión de la literatura Deep dermal fungal Infections : Review of the literature. Rev ciencias la Salud. 2018;2:145-61.

8. Zurabián $R$, Hernández Hernández $F$. Esporotricosis: la micosis más frecuente en México. Rev la Fac Med. 2019;62(5):48-55.

9. Arnaldo Benjamín Feliciano Aldama Caballero , Fabián Aldama I, Centurión, José Pereira, Vanessa Brito de Sousa Rabello RMZ-O. Esporotricosis cutánea diseminada. J Chem Inf Model. 2017;110(9):1689-99.

10. Salazar-madrigal K, Araujo-rodríguez NM, Mansilla-arévalo JJ, Leonel E, Riley G. Esporotricosis infantil : comunicación de un caso con excelente respuesta clínica al yoduro de potasio. Dermatología Rev Mex. 2018;62(3):24751.

11. Aguilar Fernández G, Araújo López PV. Micosis y nocardiosis de implantación: esporotricosis, cromoblastomicosis, micetomas y nocardiosis. Rev Nac. 2020;12(1):1-13.

12. Bertera A, Rossi MV, Tessadro G, Aloise I. Esporotricosis linfocutánea secundaria a una mordedura de gato. Dermatología Argentina. 2017;23:133-5.

13. Puebla-miranda $M$, Vásquez-ramírez $M$, González-ibarra M, Torres-lópez IH. Esporotricosis. Reporte de un caso ocupacional. Rev Hosp Juarez México. 2018;85(4):246-50.

14. Mayorga J, Fafutis-Morris M, Tlacuilo-Parra A, Guillén-Vargas C. Expresión de receptores tipo Toll 2 y 4 en macrófagos de piel de pacientes con esporotricosis cutánea. Dermatologia Rev Mex. 2015;59(1):3-8.

15. Fernández $C$, Camejo NR, González JC, Montesino M, Apaulasa K. Esporotricosis . A propósito de un caso. Rev Cubana Med Trop. 2016;68(2):171-8. 
Esporotricosis: la micosis subcutánea más distribuida en el mundo Dr. José Roberto Hernández Chacón, Dra. Sheirys Arleth Torres Morales, Dra. Evelyn María Hernández Chacón

16. Jaldín JP, Rodríguez Auad JP. Esporotricosis:

presentación de un caso inusual. Gac Medica

Boliv. 2020;43(1):95-6. 